\title{
Points to consider for reporting of germline variation in patients undergoing tumor testing: a statement of the American College of Medical Genetics and Genomics (ACMG)
}

\author{
Marilyn M. Li, MD¹, Elizabeth Chao, MD, PhD², Edward D. Esplin, MD, PhD, David T. Miller, MD, PhD ${ }^{4}$, \\ Katherine L. Nathanson, MD ${ }^{5}$, Sharon E. Plon, MD, PhD ${ }^{6,7}$, Maren T. Scheuner, MD, MPH ${ }^{8,9}$ and \\ Douglas R. Stewart, MD ${ }^{10}$; ACMG Professional Practice and Guidelines Committee ${ }^{11}$
}

Disclaimer: This statement is designed primarily as an educational resource for medical geneticists and other clinicians to help them provide quality medical services. Adherence to this statement is completely voluntary and does not necessarily assure a successful medical outcome. This statement should not be considered inclusive of all proper procedures and tests or exclusive of other procedures and tests that are reasonably directed to obtaining the same results. In determining the propriety of any specific procedure or test, the clinician should apply his or her own professional judgment to the specific clinical circumstances presented by the individual patient or specimen.

Clinicians are encouraged to document the reasons for the use of a particular procedure or test, whether or not it is in conformance with this statement. Clinicians also are advised to take notice of the date this statement was adopted, and to consider other medical and scientific information that becomes available after that date. It also would be prudent to consider whether intellectual property interests may restrict the performance of certain tests and other procedures.

Keywords: presumed germline pathogenic variants (PGPV); somatic variants; tumor testing; next-generation sequencing (NGS); secondary findings
Genetics in Medicine (2020) 22:1142-1148; https://doi.org/10.1038/s41436020-0783-8

\section{INTRODUCTION}

The sequencing of tumor-derived DNA to identify tumorspecific variations (biomarkers) with potential diagnostic, prognostic, or predictive therapeutic implications (hereafter, "tumor testing") is a prominent example of precision medicine. Although the primary goal of this testing is the identification of biomarkers to guide patient management, testing tumor genomes also has the potential to uncover clinically relevant germline variation that is associated with heritable cancer susceptibility and other conditions, and carrier status for autosomal recessive disorders, if confirmed to be present in the germline. Germline findings from tumor testing may be unexpected since many individuals harboring clinically actionable germline variants do not meet clinical guidelines for genetic testing based on personal or family history criteria. ${ }^{1-5}$
Current tumor testing strategies include tumor-only testing, tumor-normal paired testing with germline variant subtraction, and tumor-normal paired testing with explicit analysis of a group of genes associated with germline cancer predisposition. The advantages of tumor-normal paired testing include improving the somatic detection rate and streamlining results interpretation for both the tumor and germline tests. However, with tumor-only testing, germline status of variants may be inferred and thus must be confirmed with follow-up germline testing. Identifying germline pathogenic variants can inform future cancer risks, cancer surveillance, and prevention options for the patient and family members. In addition, germline genetic information, independent of somatic variation, can influence the choice of targeted therapy for a tumor. For example, Mandelker et al. identified germline variants in 38 of 1040 cancer patients (3.7\%) for whom treatment was

\footnotetext{
${ }^{1}$ Children's Hospital of Philadelphia, University of Pennsylvania Perelman School of Medicine, Philadelphia, PA, USA; ${ }^{2}$ Division of Genetic and Genomic Medicine, University of California, Irvine, School of Medicine, Irvine, CA, USA; ${ }^{3}$ Invitae, San Francisco, CA, USA; ${ }^{4}$ Division of Genetics and Genomics, Boston Children's Hospital, Department of Pathology, Brigham and Women's Hospital, Boston, MA, USA; ${ }^{5}$ Department of Medicine, Translational Medicine and Human Genetics, Perelman School of Medicine, University of Pennsylvania, Philadelphia, PA, USA; ${ }^{6}$ Baylor College of Medicine, Houston, TX, USA; ${ }^{7}$ Texas Children's Hospital, Houston, TX, USA; ${ }^{8}$ Division of Medical Genetics, Department of Pediatrics and Division of Hematology-Oncology, Department of Medicine, UCSF School of Medicine, San Francisco, CA, USA; ${ }^{9}$ Clinical Genetics Program, San Francisco VA Health Care System, San Francisco, CA, USA; ${ }^{10}$ Clinical Genetics Branch, Division of Cancer Epidemiology and Genetics, National Cancer Institute, Rockville, MD, USA;

${ }^{11}$ American College of Medical Genetics and Genomics, Bethesda, MD, USA. Correspondence: ACMG (documents@acmg.net)

The Board of Directors of the American College of Medical Genetics and Genomics approved this position statement on 24 February 2020.
} 
Table 1 Common tumor testing methods.

\begin{tabular}{|c|c|c|c|}
\hline Testing method & Sample required & $\begin{array}{l}\text { PGPV covered in } \\
\text { the test }\end{array}$ & $\begin{array}{l}\text { Confirmatory germline } \\
\text { testing }\end{array}$ \\
\hline Tumor-only testing & Tumor specimen & May be inferred & Yes \\
\hline Tumor-normal paired testing with germline variant subtraction & $\begin{array}{l}\text { Both tumor and } \\
\text { nontumor specimens }\end{array}$ & Masked & Unlikely \\
\hline $\begin{array}{l}\text { Tumor-normal paired testing with a set of cancer predisposition genes } \\
\text { in germline data deliberately analyzed }\end{array}$ & $\begin{array}{l}\text { Both tumor and } \\
\text { nontumor specimens }\end{array}$ & $\begin{array}{l}\text { Detected based on } \\
\text { test design }\end{array}$ & $\begin{array}{l}\text { No if germline results validated } \\
\text { as a germline test }\end{array}$ \\
\hline
\end{tabular}

PGPV presumed germline pathogenic variant.

modified or could be changed as a result of the germline findings. ${ }^{5}$

There may be challenges in returning germline results. Distress and anxiety, especially in the short term, have been reported in female breast cancer patients learning of a germline pathogenic variant during post-test counseling. ${ }^{6}$ This anxiety was higher among patients diagnosed within 1 year compared with those tested over a year from diagnosis; ${ }^{7}$ other studies of disclosure of genetic risk for breast cancer and other adult-onset disorders (e.g., Alzheimer disease) have not found evidence of distress and anxiety from such disclosure. ${ }^{8,9}$ Annotation of somatic tumor variation is largely focused on identification of therapeutic targets. Thus, in the germline, a variant may be of uncertain significance but, if arising as a somatic variant, may be highly actionable; the reverse is also true. These critical distinctions may be confusing to patients. Potential challenges to the oncology team may also arise from lack of familiarity with multidisciplinary guidelines informing management of germline findings, lack of expertise and experience in results interpretation, and a limited genetics workforce available to provide consultation and pre- and post-test genetic counseling. ${ }^{10,11}$ At this time, germline analysis in tumor-normal paired testing is not reimbursed (or is minimally reimbursed) due, in part, to a lack of published evidence on improved outcomes. Laboratories that provide germline results (apart from funded research studies) are thus typically required to absorb the costs of germline testing, which thus may be a disincentive to do such testing.

Currently, there is insufficient evidence to inform "best practices" for reporting presumed germline pathogenic variants (PGPVs) when tumor testing is performed. Therefore, clinical decision-making largely relies on expert opinion and experience. ${ }^{12}$ This American College of Medical Genetics and Genomics (ACMG) points to consider document will describe the potential benefits, harms, and limitations related to reporting of germline variants identified by tumor testing and include perspectives for laboratory and clinical genetics professionals. The document is based on the professional expertise and experience of the working group members, and review of relevant literature and information resources. The document is divided into three sections: (1) points to consider for clinical laboratory professionals provides an overview of different testing approaches, describes features suggestive of germline variants, and identifies issues associated with germline variant confirmation, interpretation, and reporting; (2) points to consider for clinical professionals covers tumor test selection, consenting, reporting, and genetic counseling from the clinical perspective; and (3) case examples illustrate important issues that may pose challenges, and potential solutions are offered. We do not address the use of circulating tumor DNA or germline testing alone used solely to inform therapeutic options.

\section{POINTS TO CONSIDER FOR CLINICAL LABORATORY PROFESSIONALS}

\section{Testing approaches}

Three testing approaches are routinely used: tumor-only testing, tumor-normal paired testing with germline variant subtraction, and a hybrid approach of tumor-normal paired testing with explicit analysis of a list of genes known a priori to be associated with germline cancer predisposition (Table 1). In the tumor-normal paired testing with germline variant subtraction model, variants identified in the germline tissue (typically DNA from blood or saliva) are subtracted from the variants identified in the tumor tissue. This subtraction facilitates somatic variant detection and analysis but masks any PGPV; however, germline data may be purposefully analyzed for PGPVs at the referring physician's request. Importantly, even with tumor-only testing, PGPVs can often be inferred.

Of the three common tumor testing approaches, tumoronly testing for a panel of genes is currently the most common, in large part due to the associated reduced cost for this approach relative to the others. However, tumor-normal paired testing offers greater accuracy in detecting somatic variants, as well as the potential to identify PGPVs from germline sequencing using laboratory validated testing to facilitate return of results. ${ }^{13,14}$ The paired tumor-normal model also is helpful in increasing the accuracy of secondary analyses. If tumor-normal testing is performed, and germline variants are subtracted, there is the potential risk of removing PGPVs that, were they known, might influence therapeutic selection (e.g., germline pathogenic variant in BRCA1 or $B R C A 2$ and eligibility for poly [ADP-ribose] polymerase [PARP] inhibitor treatment). There is increasing evidence that a larger proportion of cancer types are associated with germline variation than was previously recognized, such as renal cancer associated with pathogenic variants in succinate 
dehydrogenase genes. ${ }^{15}$ Thus, evidence is emerging in support of an analysis pipeline for tumor testing (or tumor-normal subtraction) that is designed to recognize PGPVs.

Nevertheless, it is important to point out that tumornormal paired testing is not a replacement for dedicated germline genetic testing since not all PGPVs will be identified with this approach. Somatic-focused bioinformatics pipelines designed to subtract germline variants make pathogenic germline variants invisible. Many tumor-normal paired testing strategies are not designed to detect copy-number variation $(\mathrm{CNV})$, leading to missed germline pathogenic CNVs. Some laboratories may opt to avoid evaluating germline variants when the purpose of the testing is to identify somatic variation from the tumor, or they may lack the expertise and/or resources to confirm and interpret PGPVs. Tumor panels often focus on genes related to diagnosis and/or treatment and may not include all susceptibility genes for that tumor type. Each laboratory should clearly describe its testing spectrum and limitations to avoid false expectations/understanding from referring physicians.

There are also important issues to consider in selecting the normal tissue for paired tumor-normal analyses. The DNA from normal tissue is typically derived from any nontumor tissues. However, the use of normal tissue adjacent to tumor can lead to missed somatic variants due to the long-known, poorly understood "field effect", in which tumor variants are found in the adjacent histologically normal tissue. ${ }^{16}$ Clonal hematopoiesis of indeterminate potential (CHIP) and aberrant clonal expansion (ACE) are two similar and recently recognized phenomena that can result in false-positive results in germline testing from blood. Both are associated with aging or chemotherapy and may be uncovered through tumor testing. CHIP involves somatic clonal variants in genes recurrently mutated in hematologic malignancies; it is detected in individuals who lack a known hematologic malignancy or other clonal disorder. ${ }^{17}$ ACE defines any clone of noncancerous cells harboring an acquired aberration that confers a mild proliferative advantage. ${ }^{18}$ Both CHIP and ACE are dynamic with expansions and contractions of the number of aberrant cells and have been observed in critical cancerassociated genes (e.g., TP53); their clinical significance is under debate. ${ }^{19}$ If not considered, CHIP or ACE can result in false-positive results for germline testing, or lead to missed somatic variants when variants in normal tissue are used for subtraction. Clinical laboratories should try to avoid using adjacent normal tissue in tumor-normal paired testing. Laboratories should also implement protocols to recognize and confirm CHIP and ACE and ensure appropriate followup and reporting of these postzygotic somatic variations.

\section{Features of PGPVs}

It is not straightforward to determine which variants may be germline when tumor-only sequencing is performed. The variant allele frequency (VAF) of heterozygous PGPVs is typically in the $40 \%$ to $60 \%$ range. However, the VAF of germline variants may be outside of this range for many reasons, such as tumor biology (e.g., loss of the wild-type allele through deletion or copy-number neutral loss of heterozygosity, or amplification of the mutant allele). In addition, some types of germline variants are less likely to be captured or identified due to technical limitations associated with next-generation sequencing (NGS), such as genomic rearrangements and large insertions/deletions, or expansion/ contraction of repetitive sequences (homopolymers, di- and trinucleotide repeats). As with all types of sequencing, GCrich regions and pseudogenes make true variant identification more difficult. Although the use of a VAF threshold may seem to be a convenient heuristic to rule a germline variant in or out, there are no established cutoffs that consistently and accurately indicate germline status and conversely somatic variant VAF can overlap 0.5. Thus, all PGPVs should have confirmatory germline testing. Unfortunately, insurance companies may use VAF as a metric in deciding whether to pay (or not) for confirmatory germline testing (Box 1).

In addition to VAF, several other factors warrant consideration when deciding if a variant could be a PGPV. A known founder variant in a gene associated with a cancer predisposition syndrome is almost always germline. ${ }^{5}$ Consideration of the relative frequency of somatic versus germline variation and tumor type is also important. For some genes (e.g., BRCA2, $S D H B$ ), a significant fraction of variants identified through tumor testing are germline in origin, whereas for others the vast majority are actually somatic (e.g., TP53 in adult cancers). ${ }^{5,20,21}$ Additionally, patient clinical information such as tumor type, age at onset, bilateral/ multiple versus unilateral primary cancers, and family history of cancer should be carefully reviewed when available to the testing laboratory. Tumors associated with a known cancer predisposition syndrome may carry a higher probability of a PGPV. For example, the majority (50-80\%) of children diagnosed with adrenocortical carcinoma have a germline TP53 pathogenic variant (Li-Fraumeni syndrome); patients with uveal melanoma often $(36.2 \%)$ carry germline null pathogenic variants in $B A P 1 . .^{22,23}$ In some situations, tumor-

Box 1 A 7-year-old child was diagnosed with a rhabdomyosarcoma of the jaw.

A pediatric-focused tumor variant panel (tumor-only analysis) was performed and revealed a TP53 missense variant [NM_000546.5(TP53): c.524G $>$ A (p.Arg175His)]. This variant is well-described in COSMIC, ClinVar, and the International Agency for Research on Cancer (IARC) TP53 database as both a somatic and germline variant, with the latter in patients with Li-Fraumeni syndrome. There was no significant maternal family history of cancer but there was little information on the paternal side. The patient was referred to a cancer genetics clinic for germline validation. Preauthorization for TP53 testing (including limited variant testing) was denied through two rounds of appeal. In the first round, authorization was denied since the child did not meet Chompret criteria. In the second round of appeal, authorization was denied since the confirmation of somatic variants requires a variant fraction of 0.5 or higher (the variant in the patient was at 0.475 ). The lack of germline validation is impeding determination of whether this child (or any at-risk siblings) might benefit from the Li-Fraumeni syndrome ("Toronto Protocol") surveillance regimen. 
specific factors may increase or decrease the probability of a particular germline finding. For example, somatic $\beta$-catenin variants make finding a germline $A P C$ variant less likely. ${ }^{24,25}$ Younger-than-typical age at onset may suggest an underlying cancer predisposition, such as very early onset breast cancer. ${ }^{26}$ For patients with bilateral/multiple primary tumors, variants found in associated genes, such as $\mathrm{MSH} 2$ in patients with colon and endometrial cancer (even without early onset), are more likely germline. ${ }^{27}$ A family history of cancer may suggest an inherited germline pathogenic variant in the family; however, a negative family history does not rule out a cancer predisposition syndrome due to incomplete penetrance, de novo variants, misattributed paternity, or lack of knowledge of family history. Unfortunately, family history is often not available to laboratory professionals at the time of testing. Laboratories may establish a list of cancer syndromes and associated genes included in the regions of interest of their tumor testing to facilitate the identification of PGPVs.

\section{Reporting of PGPV}

In tumor-only testing, reporting PGPVs associated with cancer predisposition should be considered, along with recommendations for genetic consultation and follow-up confirmatory germline testing. For laboratories with limited resources or expertise in germline variant analysis and reporting, it should be clearly stated on the report that only somatic variation was evaluated. The report should also state whether germline variants have been subtracted in tumornormal paired testing.

There are multiple different mechanisms for returning genomic test results to referring physicians and patients. Most laboratories in academic institutions return results only to the referring physicians through internal medical record systems and discuss challenging cases at tumor conferences and/or molecular tumor boards. Although there is a range of practices, third-party laboratories often return the results to the client institutions with little physician-laboratory interaction. Consequently, PGPVs could be overlooked unless specifically noted on the report. Genomic testing results may be automatically entered to the patient medical record, creating opportunities for patients or other health-care providers to access their results prior to a referring physician's review, potentially leading to unwanted confusion. We recommend that genomic test results should be delivered to patients by qualified health-care professionals to maximize their understanding of the test results and to avoid potential confusion or harm.

\section{Points to consider for clinical laboratory professionals}

- There are three tumor testing strategies: tumor-only testing, tumor-normal paired testing with germline variant subtraction, or tumor-normal paired testing with full analysis of the germline data from a subset of genes associated with cancer predisposition.

- Tumor-normal paired testing is not a substitute for dedicated germline testing unless the germline application was designed, validated, and implemented as part of the tumor-normal paired testing protocol.

- A known founder variant in a cancer predisposition gene detected on tumor-only testing is almost always germline, but still merits orthogonal confirmation.

- Copy-number variation and variant characteristics such as large indels or homopolymers may affect variant allele frequencies and may require specialized testing methods to report.

- Clinical data such as tumor type, age at cancer onset, bilateral or multiple tumors, and family history of cancer can help inform the evaluation of PGPVs.

- Using "normal" adjacent tissue in tumor-normal paired testing should be discouraged to avoid the risk of false positives/negatives due to field "cancerization" effects.

- Clonal hematopoiesis of indeterminate potential (CHIP) and aberrant clonal expansion (ACE) should be factored into genomic analyses, to minimize false-positive germline results or false-negative somatic results.

\section{POINTS TO CONSIDER FOR CLINICAL PROFESSIONALS}

\section{Pretest considerations}

When ordering tumor testing, the clinician should consider the likelihood of an underlying cancer predisposition syndrome. Clinical indicators for germline cancer predisposition include multiple primary cancers in an individual, cancer presentation in unusual locations or at younger-than-usual ages, rare cancers, specific pediatric cancers (e.g., retinoblastoma), the presence of a cancer family history, or unusual findings or comorbidities (e.g., dysmorphic features, developmental delay, congenital heart disease). ${ }^{1,28}$ Clinically actionable variation in the germline may be missed even on tumor-normal paired testing. Thus, if there is an indication, dedicated germline testing should be offered to patients, following appropriate genetic counseling. Additionally, suspected germline findings identified on tumor testing needs germline confirmation testing on a second, independent sample.

The ordering clinician should also understand what genes are (and are not) included and the general technical limitations of the genetic testing of a region of interest (ROI). In particular, it should be understood if evaluation of copy-number variation (e.g., deletions and duplications) is included as part of the test. CNV determination may be performed if no pathogenic sequence variation is identified; in addition, a germline PGPV may be masked through a somatic deletion. Increasingly, copy-number assessment is conducted using NGS data; however, the assay and analytic performance can vary between laboratories. When in doubt, as with any variant, confirmation with an orthogonal method (e.g., microarray or polymerase chain reaction [PCR]-based methods, like multiplex ligation-dependent probe amplification [MLPA]) is indicated. The ordering clinician should also understand that for some genes (like MSH2), germline 
pathogenicity may arise from an inversion event not detected on most platforms rather than sequence or copy-number alteration.

\section{Patient education and informed consent}

Clinicians should inform patients that tumor testing, regardless of the testing strategy, may unexpectedly identify a PGPV, which if confirmed to be present in the germline, could have significant implications for their management and family. ${ }^{29}$ In tumor-normal paired testing, informed consent should be obtained if germline results will be disclosed. The consenting process should include an option to opt out of germline reporting although germline inference may still unexpectedly occur. The informed consent should outline the potential benefits, harms, and limitations of learning about a germline result. Potential benefits of identifying a PGPV include better risk counseling and family planning, identification of other family members through cascade testing, and gene- and syndrome-specific medical surveillance. Germline results may also influence treatment decisions. For example, use of PARP inhibitors for breast and/or ovarian cancer patients with germline $B R C A 1 / B R C A 2$ pathogenic variants, or PD-1 inhibitors for Lynch syndrome patients with advanced cancers of any type (Box 2). ${ }^{30-32}$ Identification of a PGPV may be an entry point for genetic counseling for a family and permits cascade testing and identification of family members at risk (Box 3).

The informed consent process may be done using traditional models (e.g., in person, or by phone with the ordering provider) or by using innovative methods (e.g., online videos, validated artificial intelligence-based methods such as chatbots). Example language to describe the possibility of identifying a PGPV: "Tumor testing may uncover DNA changes (variants) that increase your risk for cancer. Such findings may be inherited and could have health implications for you and your family. If found, your doctor may refer you for additional testing and genetic counseling. You have the right to opt out and not learn about these changes. A discussion with a geneticist or genetic counselor, health-care professionals skilled in explaining genetics results, may help you better understand these options." It should be made clear to patients that they have the right to opt out of learning about PGPVs identified in their tumor. Nongenetics clinicians may consider enlisting genetics professionals to address questions patients may have regarding PGPVs. Lastly, the informed consent process should be documented in the patient chart and on the test requisition form.

In addition to identifying unexpected PGPVs in cancer predisposition genes, secondary or incidental genetic findings may also be uncovered (Box 4). These include variants in the 59 genes on the current ACMG Secondary Finding v2.0 list. ${ }^{33}$ There are more than 20 hereditary cancer genes on this list, which are frequently included when tumor testing is performed. Larger panel or exome/genome sequencing may also reveal variations in other genes that underlie other diagnoses in the tested individual (e.g., a variant in a
Box 2 A 45-year-old male presented to his primary care physician with history of change in stool pattern and an athome fecal occult blood test positive three times over the past month.

At initial evaluation, there was no reported family history of colorectal cancer. Subsequent colonoscopy revealed colonic adenocarcinoma, which additional work-up indicated is metastatic. Tumor-only somatic analysis identified a single pathogenic BRCA2 variant (NM_000059.3[BRCA2]: c.3975_3978dup [p.Ala1327fs*4]). Family history discovered no evidence of breast or ovarian cancer. Follow-up germline genetic testing proved the $B R C A 2$ variant to be of germline origin and ruled out the presence of pathogenic variants in canonical colorectal cancer genes. The unexpected $B R C A 2$ germline variant in this colorectal cancer patient prompted genetic counseling and cascade familial testing for his three younger sisters, warranted initiation of prostate cancer screening for the patient, and qualified the patient to be enrolled in a PARP inhibitor clinical treatment trial (NCT02286687), consistent with National Comprehensive Cancer Network (NCCN) recommendations.

Box 3 A 23-year-old woman developed nonspecific abdominal pain and progressive hirsutism.

She had been otherwise healthy. Although there was no significant family history of cancer, her father had a thyroidectomy for symptomatic multinodular goiter at age 35 years. Evaluation of the woman revealed elevated serum testosterone and a right ovarian mass. A presumptive diagnosis of Sertoli-Leydig cell tumor was made. Sequencing of the right ovarian mass revealed a pathogenic frameshift variant (NM_030621.4 [DICER1]:c.5469del [p.Ser1823Argfs*15]) and a hotspot variant (NM_030621.4[DICER1]:c.5429A>T [p.Asp1810Val]) in DICER1. Review of sequencing from a DNA sample from the patient's blood revealed that the p.Ser1823fs variant was germline. Cascade genetic testing in the family showed that her 15-year-old sister, father, and paternal aunt all harbored the germline variant and DICER1 surveillance guidelines were implemented.

Box 4 A 17-year-old previously healthy male without a significant family cancer history developed a Ewing sarcoma.

Tumor-only DNA- and RNA-based panel testing revealed an EWSR1/ FLI1 fusion, which results in a well-known fusion protein associated with Ewing sarcoma. A pathogenic variant in BRCA1 (NM_007294.3[BRCA1]: c.5266dupC [p.Gln1756Profs*74]; VAF: 47\%), a known founder variant in Ashkenazi Jews, was also identified. The BRCA1 variant was confirmed in a germline sample. Cascade testing showed that the patient's 25 -yearold sister also harbored the variant, prompting a referral to a cancer genetics clinic.

chromatin remodeling gene in a person with cancer and history of developmental delay). The larger the number of interrogated genes (panel, exome, genome) the more numerous and complex the possible findings.

Ideally, the pretesting discussion should also review insurance coverage issues. The confirmatory testing and clinical follow-up of PGPVs (whether primary, secondary, or incidental) may not be covered by insurance. Insurance companies, states, and Medicare/Medicaid may have specific policies and/or regulations on germline confirmation that can be limited to only a few genes or very specific clinical scenarios (Box 1). 


\section{Disclosure of results}

The basic principles of medicine apply to genetic testing: when ordering a test that might reveal a germline component, the clinician (and patient, through the pretesting education process) need to be ready to receive and act on the results. This is especially true in the context of rapid result return through patient portals. Oncologists and surgeons ordering these tests should consider establishing a referral network with cancer genetics expertise (e.g., clinical geneticist or genetic counselor) to help manage PGPVs. Doing so should facilitate more timely referrals, alleviate patient anxiety, and avoid unneeded follow-up procedures or evaluations. Cancer genetics professionals can also provide guidance on test result interpretation.

\section{Points to consider for clinical professionals}

- Individuals undergoing tumor testing should undergo informed consent of the possibility that a PGPV might be discovered. However, if there is clinical indicator for germline cancer predisposition, then dedicated germline testing should be ordered.

- Patient choice and autonomy (opt-out of PGPV result return) should be respected.

- When automated methods are used for pre- and posttesting education and counseling, clinicians with experience in cancer genetics should be available to answer specific questions.

- Patients should be informed that discovery of a PGPV would prompt referral for genetic consultation and the possibility of confirmatory germline testing.

- Confirmatory germline testing should be performed in a clinical laboratory that has adequate resources and expertise in conducting germline testing and interpreting and reporting the test results.

- Positive germline test results should be returned by qualified and experienced clinicians (e.g., oncologists with genetics expertise, geneticists, and genetic counselors).

\section{SUMMARY AND FUTURE DIRECTIONS}

Tumor testing is emerging as the first choice to identify biomarkers that inform precision cancer care. Surprisingly, studies have shown that more than $50 \%$ of germline PGPVs identified with tumor testing would have been missed if germline testing had been limited to guideline-concordant care. $^{4,5}$ The identification of PGPVs may have profound health and reproductive implications for the cancer patient and their family, and may identify therapeutic targets (e.g., PARP inhibitors for BRCA1 or BRCA2 carriers). Thus, individuals undergoing tumor testing should be informed prior to testing that a germline variant may be uncovered, and not when results are returned. PGPVs should be carefully evaluated, confirmed, and reported when tumor testing is performed. Currently, there is a lack of evidence for best practices to report PGPVs to patients who want them. The development of practice guidelines related to the detection and return of PGPVs should include all stakeholders, including genetics, oncology, and pathology professionals; government and private payers; and patient/disease advocacy groups. Future research is needed to generate evidence regarding patient outcomes resulting from the reporting of PGPVs with tumor testing, which will in turn inform evidence-based guidelines for selecting tumor testing, disclosing germline test results, and managing the associated disease risk for cancer patients and their family members.

\section{DISCLOSURE}

M.M.L. is on the Scientific Advisory Board of Roche Sequencing Solutions. D.R.S. performs contract clinical telegenetics work for Genome Medical, Inc. E.D.E. is a full-time employee of Invitae. E.C.C. is currently employed by Ambry Genetics. S.E.P. is a member of the scientific advisory board of Baylor Genetics Laboratories. The other authors declare no conflicts of interest.

Publisher's note Springer Nature remains neutral with regard to jurisdictional claims in published maps and institutional affiliations.

\section{REFERENCES}

1. Hampel $H$, Bennett $R L$, Buchanan $A$, et al. A practice guideline from the American College of Medical Genetics and Genomics and the National Society of Genetic Counselors: referral indications for cancer predisposition assessment. Genet Med. 2015;17:70-87.

2. Beitsch PD, Whitworth PW, Hughes K, et al. Underdiagnosis of hereditary breast cancer: are genetic testing guidelines a tool or an obstacle? J Clin Oncol. 2019;37:453-460.

3. Nicolosi P, Ledet E, Yang S, et al. Prevalence of germline variants in prostate cancer and implications for current genetic testing guidelines. JAMA Oncol. 2019;5:523-528.

4. Zhang J, Walsh MF, Wu G, et al. Germline mutations in predisposition genes in pediatric cancer. N Engl J Med. 2015;373:2336-2346.

5. Mandelker D, Zhang L, Kemel Y, et al. Mutation detection in patients with advanced cancer by universal sequencing of cancer-related genes in tumor and normal DNA vs guideline-based germline testing. JAMA. 2017;318:825-835

6. Watson $M$, Foster $C$, Eeles $R$, et al. Psychosocial impact of breast/ovarian (BRCA 1/2) cancer-predictive genetic testing in a UK multi-centre clinical cohort. Br J Cancer. 2004;91:1787-1794.

7. Jacobs $C$, Patch $C$, Michie $S$. Communication about genetic testing with breast and ovarian cancer patients: a scoping review. Eur J Hum Genet. 2019;27:511-524.

8. Roberts JS, Christensen KD, Green RC. Using Alzheimer's disease as a model for genetic risk disclosure: implications for personal genomics. Clin Genet. 2011;80:407-414.

9. Schwartz MD, Peshkin BN, Hughes C, Main D, Isaacs C, Lerman C. Impact of BRCA1/BRCA2 mutation testing on psychologic distress in a clinicbased sample. J Clin Oncol. 2002;20:514-520.

10. Bernhardt BA, Silver R, Rushton CH, Micco E, Geller G. What keeps you up at night? Genetics professionals' distressing experiences in patient care. Genet Med. 2010;12:289-297.

11. Talwar D, Tseng TS, Foster M, XU L, Chen LS. Genetics/genomics education for nongenetic health professionals: a systematic literature review. Genet Med. 2017;19:725-732.

12. DeLeonardis K, Hogan L, Cannistra SA, Rangachari D, Tung N. When should tumor genomic profiling prompt consideration of germline testing? J Oncol Pract. 2019;15:465-473.

13. Parsons DW, Roy A, Yang Y, et al. Diagnostic yield of clinical tumor and germline whole-exome sequencing for children with solid tumors. JAMA Oncol. 2016;2:616-624.

14. AlDubayan SH. Leveraging clinical tumor-profiling programs to achieve comprehensive germline-inclusive precision cancer medicine. JCO Precis Oncol. 2019 May 23; https://doi.org/10.1200/PO.19.00108 [Epub ahead of print]. 
15. Wang G, Rao P. Succinate dehydrogenase-deficient renal cell carcinoma: a short review. Arch Pathol Lab Med. 2018;142:1284-1288.

16. Chai $H$, Brown RE. Field effect in cancer-an update. Ann Clin Lab Sci. 2009;39:331-337.

17. Steensma DP, Bejar R, Jaiswal $S$, et al. Clonal hematopoiesis of indeterminate potential and its distinction from myelodysplastic syndromes. Blood. 2015;126:9-16.

18. Forsberg LA, Gisselsson D, Dumanski JP. Mosaicism in health and disease —clones picking up speed. Nat Rev Genet. 2017;18:128-142.

19. Weitzel JN, Chao EC, Nehoray B, et al. Somatic TP53 variants frequently confound germ-line testing results. Genet Med. 2018;20:809-816.

20. Riaz N, Blecua P, Lim RS, et al. Pan-cancer analysis of bi-allelic alterations in homologous recombination DNA repair genes. Nat Commun. 2017;8:857.

21. Shen $\mathrm{H}$, Shih J, Hollern DP, et al. Integrated molecular characterization of testicular germ cell tumors. Cell Rep. 2018;23:3392-3406.

22. Petr EJ, Else T. Adrenocortical carcinoma (ACC): when and why should we consider germline testing? Presse Med. 2018;47(7-8 Pt 2): e119-e125.

23. Walpole S, Pritchard AL, Cebulla CM, et al. Comprehensive study of the clinical phenotype of germline BAP1 variant-carrying families worldwide. J Natl Cancer Inst. 2018;110:1328-1341.

24. Wang WL, Nero C, Pappo A, Lev D, Lazar AJ, Lopez-Terrada D. CTNNB1 genotyping and APC screening in pediatric desmoid tumors: a proposed algorithm. Pediatr Dev Pathol. 2012;15:361-367.

25. Dubbink HJ, Hollink I, Avenca Valente $C$, et al. A novel tissue-based $\beta$ catenin gene and immunohistochemical analysis to exclude familial adenomatous polyposis among children with hepatoblastoma tumors. Pediatr Blood Cancer. 2018;65:e26991.
26. Aloraifi $\mathrm{F}$, Alshehhi $\mathrm{M}, \mathrm{McD}$ evitt $\mathrm{T}$, et al. Phenotypic analysis of familial breast cancer: comparison of BRCAx tumors with BRCA1-, BRCA2carriers and non-familial breast cancer. Eur J Surg Oncol. 2015;41: 641-646.

27. Hegde M, Ferber M, Mao R, et al. ACMG technical standards and guidelines for genetic testing for inherited colorectal cancer (Lynch syndrome, familial adenomatous polyposis, and MYH-associated polyposis). Genet Med. 2014;16:101-116.

28. Bashford MT, Kohlman W, Everett J, Parrott A, Pollin TI. Addendum: a practice guideline from the American College of Medical Genetics and Genomics and the National Society of Genetic Counselors: referral indications for cancer predisposition assessment. Genet Med. 2019; 21:2844.

29. Raymond VM, Gray SW, Roychowdhury S, et al. Germline findings in tumor-only sequencing: points to consider for clinicians and laboratories. J Natl Cancer Inst. 2015;108:djv351.

30. Robson M, Im SA, Senkus E, et al. Olaparib for metastatic breast cancer in patients with a germline BRCA mutation. N Engl J Med. 2017;377: 523-533.

31. Litton JK, Rugo HS, Ettl J, et al. Talazoparib in patients with advanced breast cancer and a germline BRCA mutation. N Engl J Med. 2018;379:753-763.

32. Overman MJ, Lonardi S, Wong KYM, et al. Durable clinical benefit with nivolumab plus ipilimumab in DNA mismatch repair-deficient/ microsatellite instability-high metastatic colorectal cancer. J Clin Oncol. 2018:36:773-779

33. Kalia SS, Adelman K, Bale SJ, et al. Recommendations for reporting of secondary findings in clinical exome and genome sequencing, 2016 update (ACMG SF v2.0): a policy statement of the American College of Medical Genetics and Genomics. Genet Med. 2017;19:249-255. 